\title{
Serous cystadenocarcinoma of the pancreas: report of a case and management reflections
}

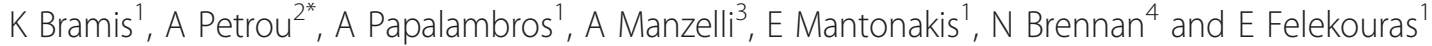

\begin{abstract}
Background: Serous adenomas represent $1-2 \%$ of pancreatic neoplasms and typically are asymptomatic not requiring any treatment and simple observation is the option of choice. Although, they carry a realistic risk of malignancy despite the general view that they never become malignant. We report a case, which, according to our best knowledge is the 27 th case reported in the literature.

Methods: We reviewed the literature by performing a search in Pub Med and Medline.

Results: A 86-year old patient known to have a serous cystadenoma of the pancreas treated conservatively through a close clinical and radiological follow up which was unattended for 4 years ending up to our emergency department suffering an acute abdomen. Exploratory laparotomy revealed a perforated prepyloric ulcer which was treated accordingly. Patient died some weeks later due to severe medical co morbidities.

Conclusion: Serous cystic neoplasms of the pancreas carry a realistic risk of malignancy despite the general view that they never become malignant. In our opinion the treatment strategy of serous cystic neoplasms of the pancreas should be aggressive even in cases of remote metastases since prognosis of the disease is satisfactory
\end{abstract}

Keywords: pancreatic cancer, cystic tumors of the pancreas, serous cystadenoma, serous cystadenocarcinoma

\section{Background}

Malignant cystic tumors of the pancreas are very rare, accounting for about $1 \%$ of all pancreatic malignancies [1,2]. Pancreatic cystic neoplasms comprise $1-2 \%$ of pancreatic lesions [3] and most of these lesions are cystadenomas[3,4]. In 1978, Compagno and Oertel [5] issued an histopathologic classification of cystic neoplasms of the pancreas identifying two different types with different biological behavior. The first type is considered the serous cystic neoplasm and the serous cystadenoma represents numerically the most common entity. Also called glycogen-rich cystadenoma or microcystic adenoma is generally considered to be a benign condition although serous cystadenocarcinoma is a rare but known malignant condition described in the literature. Mucinous cystic neoplasms of the pancreas are the other type of lesions and possess the more frequent ability to transform to its malignant counterpart, namely mucinous cystadenocarcinoma [6]. Today serous and mucinous pancreatic neoplasms are classified

\footnotetext{
* Correspondence: thpetrou@gmail.com

${ }^{2}$ HPB Surgery, Nicosia Surgical Department, Cyprus

Full list of author information is available at the end of the article
}

according to the WHO nomenclature as tumors of the exocrine pancreas [7].

According to the latest WHO classification these tumors are called serous cystic neoplasms, which are cystic epithelial neoplasms composed of glycogen-rich, epithelial cells that produce a watery fluid similar to serum. Most of these are benign (serous cystadenomas), and in rare cases are malignant (serous cystadenocarcinoma). Serous adenoma has four variants: macrocystic serous cystic neoplasm, solid serous neoplasm, von Hippel-Lindau (VHL)-associated serous cystic neoplasm, and mixed serous-neuroendocrine neoplasm. The term serous cystadenoma refers to microcystic type (the most frequent) of the neoplasm [4].

In 1989, George et al [8] first reported a case of serous microcystic adenoma behaving in a malignant fashion and since then, according to our best knowledge, 26 cases have been reported in the literature. According to WHO the prevalence of malignancy of serous cystic neoplasms of the pancreas is 1\%-3\% [4]. We report a case of serous cystadenocarcinoma with extensive local invasion and liver metastases.
C Biomed Central

C 2012 Bramis et al; licensee BioMed Central Ltd. This is an Open Access article distributed under the terms of the Creative Commons Attribution License (http://creativecommons.org/licenses/by/2.0), which permits unrestricted use, distribution, and reproduction in any medium, provided the original work is properly cited. 


\section{Case presentation}

An 86-year old woman presented at the emergency department suffering from severe acute upper abdominal pain. Her past medical history included hypertension, diabetes mellitus, coronary heart disease, atrial fibrillation, chronic obstructive pulmonary disease and twenty years before she had an omphalocele repair. The patient was in a close clinical and radiological follow up for monitoring a pancreatic serous cystadenoma diagnosed incidentally with a computed tomography (CT) scan 10 years earlier. The lesion was extensively studied with magnetic resonance imaging (MRI), endoscopic ultrasound (EUS), fine needle aspiration (FNA), serology and blood test screening. At that time she was addressed for a routine year follow up scan which she attended regularly for six years. Patient did not attend her follow up time table for the last four years. Upon admission to the emergency department, chest and abdominal $\mathrm{x}$-rays were performed and turned out inconclusive. Routine laboratory studies revealed marked leukocytosis, severe anemia, and blood glucose levels exceeding $500 \mathrm{mg} / \mathrm{dl}$. Due to inconclusive diagnosis, an intravenous contrast $\mathrm{CT}$ scan was ordered and revealed a large amount of free peritoneal air and free fluid. (Figure 1,2) Moreover, a left upper quadrant $17 \mathrm{~cm}$ large mass originating from the stomach was revealed along with secondary liver lesions. An urgent laparotomy showed a prepyloric ulcer perforation and a large inoperable mass arising from the pancreatic body and largely invading the posterior wall of the stomach occupying the upper abdomen with multiple hepatic secondary lesions. A liver biopsy was taken, the stomach perforation was sutured closed and a washout was performed. The histopathologic findings from the biopsy material were consisted of small and medium size cystic lesions surrounded and divided by hyalloid collagenous matrix. The cysts were lined by cuboidal epithelial cells with clear cytoplasm and round nuclei without atypical or mitotic activity as demonstrated by pathology. The glycogen content was documented by PAS positive reaction. These findings were consistent with serous microcystic adenoma of the pancreas and the malignant nature of the tumor was confirmed by the presence of the extensive invasive large inoperable pancreatic mass with hepatic metastasis. The patient after a brief period in intensive care unit for respiratory problems recovered from surgery in 10 days but died a month later because of other, unrelated, serious medical problems.

\section{Conclusions}

The prevalence of cancer among serous cystadenomas is reported to be $3 \%$ since the first case reported in the literature in 1989. It is rather clear from the literature that the differentiation between benign and malignant serous cystadenoma both histologically and clinically may be very difficult [9-11]. Additionally the presenting symptoms are not characteristic. To date serous cystadenoma is considered malignant when tumor invasion of surrounding tissues and organs or distant metastases are present [4]. According to the literature most cases of serous cystadenocarcinoma show synchronous or metachronous liver metastases, lymph node infiltration, splenic invasion, splenic vein infiltration and thrombosis, neural, perineural, and stromal invasion, as well as stomach, lung, adrenals, peritoneal, and colonic mesentery metastases [12-14]. Nowadays, the current management of serous cystadenomas of the pancreas is essentially conservative. In fact, once diagnosed correctly through CT scans, MRI, EUS, FNA, serology and blood test studies, observation and routinely reassessment of asymptomatic lesions is indicated thereby avoiding a major operation and resulting morbidity and mortality [1-3]. In our department we practice a six monthly follow-up. Nevertheless, clinicians should be aware of the possibility for malignant transformation in serous cystic neoplasms and should maintain a high index of suspicion when certain clues appear. These include the onset of new symptoms, worsening of symptoms, or rapid enlargement of the mass [15]. In these cases, resection may be indicated, despite the lack of objective evidence for malignancy obtained from preoperative imaging, endoscopy, and biopsies [3,13,15-17]. Our trust multidisciplinary specialist team board adopts and agrees with the conservative approach in the majority of the benign cases. Although, considering the chance of malignant transformation, our policy is to strictly discuss the treatment options with the patient and propose radical surgery especially in young and fit patients where the clinical and radiological situation of their lesions is minimally doubtful, unclear or borderline.

In the present case the course of the disease was indolent and only discovered after a co morbid condition requiring admission to the hospital. This case demonstrates that conservative management often does not have the success hoped for. In fact, the best results are guaranteed in those cases where patients are closely followed up and attended to their scheduled scans.

Cystic lesions of the pancreas are a heterogeneous group of disorders, newly defined, with varied biological behavior. The prevalence of such neoplasms is increasing. The reason is probably due to the spread and efficacy of diagnostic tools which frequently pick up, cystic pancreatic lesions in asymptomatic patients as incidental finding $[17,18]$. A thorough knowledge and experience on the matter by the surgical team is more than important in order to have a proper classification and treatment of these conditions. The morphological study of these tumors is based on the following investigations: ultrasound, CT scans MRI and EUS with FNA. Among this 


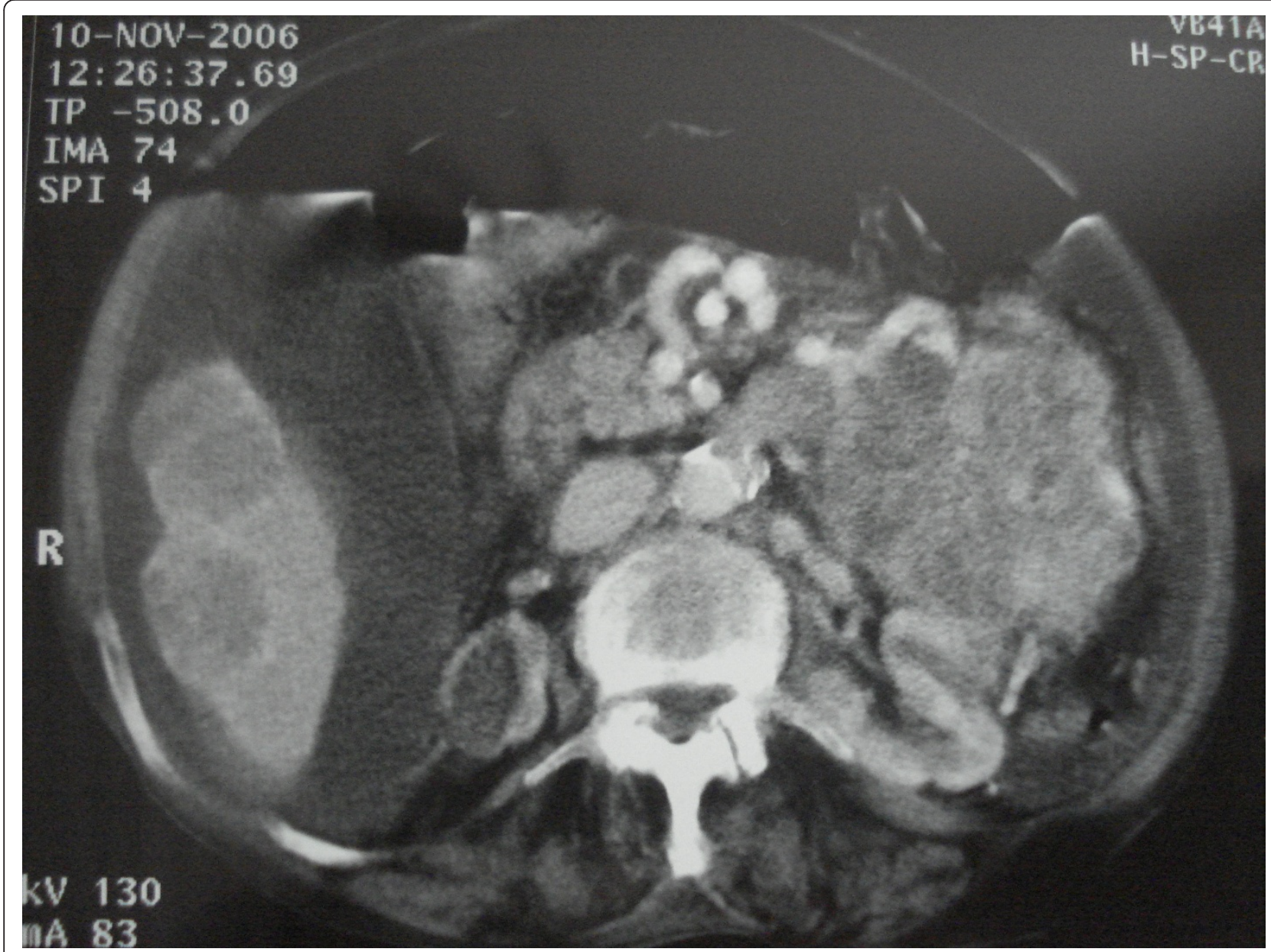

Figure 1 Large amount of free peritoneal air and free fluid alongside with evidence o metastatic liver disease

group of lesions, the serous cystadenoma is typically asymptomatic; not requiring any treatment and simple observation is the option of choice [19-21]. Serous cystic neoplasms of the pancreas although they carry a realistic risk of malignancy, the predominant view is that they never become malignant. In our opinion the treatment strategy of cystic serous neoplasms of the pancreas should be aggressive, although evidence for that is weak since it is largely based on case reports. Consequently, more studies are needed to set treatment guidelines for serous cystic neoplasms of the pancreas. Surgical treatment is considered necessary only for symptomatic patients or in cases of diagnostic doubt mainly because of the perioperative risks of radical pancreatic surgery especially when a benign disease is addressed. Whilst the management of intraductal papillary mucinous neoplasms and mucinous cystic neoplasms of the pancreas is regulated by an extended literature and even by international consensus guidelines, for the benign or borderline serous neoplasms of the pancreas a shared management vision is still needed. As mentioned, our clinical behavior has recently turned towards a greater focus on surgery. We consider that surgery of the body and especially the tail of the pancreas is technically safe and feasible with improved rates of morbidity and mortality and better impact on patient thanks to new surgical tools, better surgical materials, new technology and new advancements in surgery such as the minimally invasive access. Furthermore, pancreatic surgery is practiced in high volume centers with great experience, thus minimizing perioperative morbidity and mortality [22]. Thus, in selected patients with benign pancreatic serous cystic lesions of considerable size and a borderline clinical behavior, after a short period of clinical and radiological followup, we feel that a surgical treatment must be offered.

Considering the current numbers and the future trend of this matter, a consensus review of the management of the whole spectrum of the cystic pancreatic neoplasms is needed. Although, practice guidelines are missing, in 


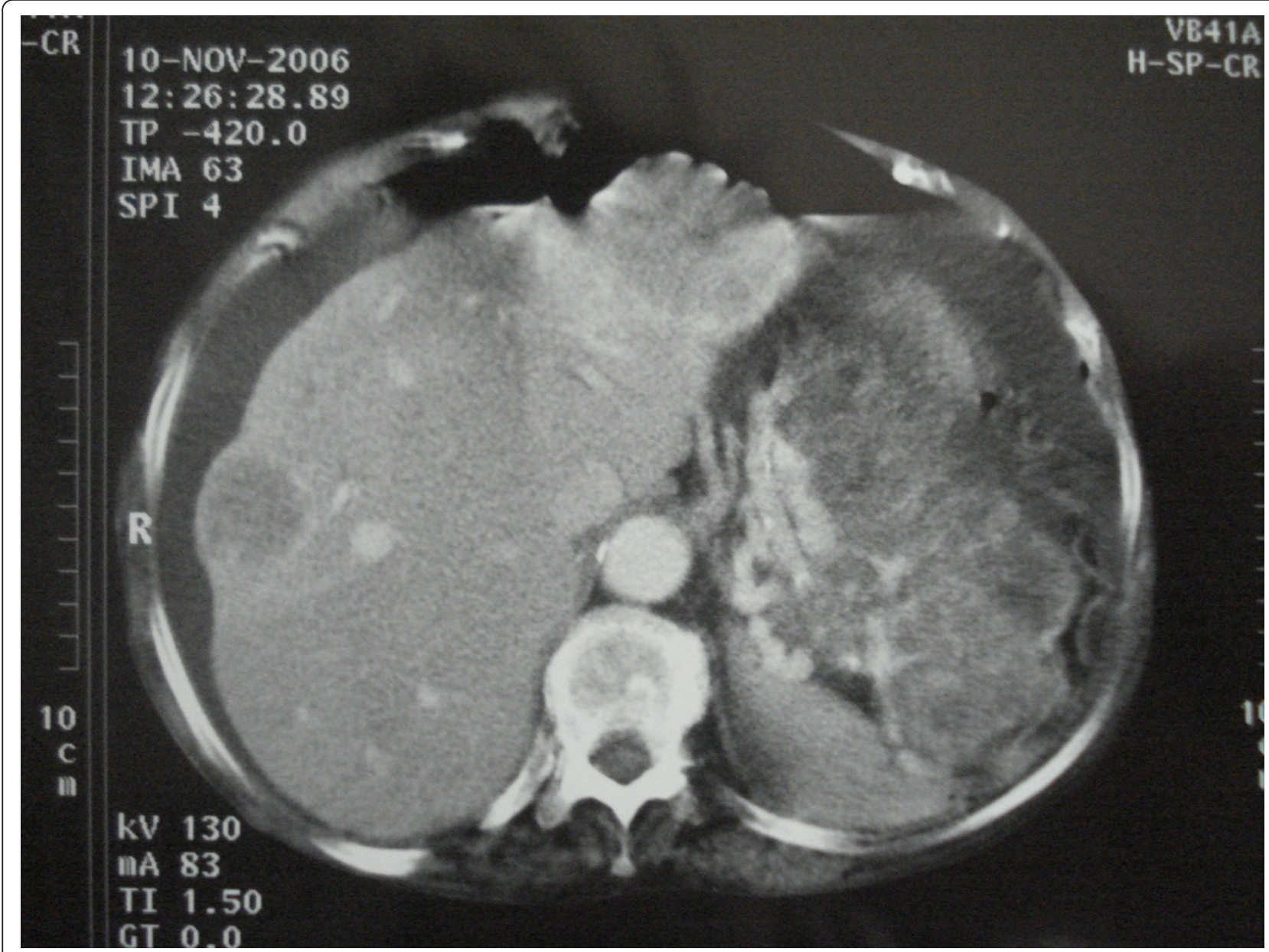

Figure 2 A left upper quadrant $17 \mathrm{~cm}$ originating from the stomach along with multiple secondary liver lesions

cases of cystic serous cystadenomas, an extensive discussion with the patients on the management options must be offered.

\section{Consent}

Written informed consent was obtained from the patient for publication of this case report and accompanying images. A copy of the written consent is available for review by the Editor-in-Chief of this journal.

\section{Author details}

'LAIKON Hospital, First Department of Surgery, University of Athens Medical School, Greece. ${ }^{2}$ HPB Surgery, Nicosia Surgical Department, Cyprus.

${ }^{3}$ Hepatobiliary and Pancreatic Surgical Department, Oxford Radcliffe Hospitals NHS Foundation Trust. ${ }^{4}$ Churchill Hospital, Oxford, UK.

\section{Authors' contributions}

$\mathrm{BK}, \mathrm{PA}, \mathrm{PA}, \mathrm{FE}$ were the surgical team involved in the management of the patient. ME performed the pathological assessment. BN and MA designed and co-wrote the paper. All authors read and approved the final manuscript.

\section{Competing interests}

The authors declare that they have no competing interests.
Received: 12 March 2011 Accepted: 8 March 2012

Published: 8 March 2012

\section{References}

1. Siech M, Tripp K, Schmidt-Rohlfing B, Mattfeldt T, Widmaier U, Gansauge F, Görich J, Beger HG: Cystic tumors of the pancreas: Diagnostic accuracy, pathologic observations and surgical consequences. Langenbecks Arch Surg 1998, 383:56-61.

2. Horvath $\mathrm{K}$, Charbot JA: An aggressive resectional approach to cystic neoplasms of the pancreas. Am J Surg 1999, 178:262-274.

3. Tseng JF, Warshaw AL, Sahani DV, Lauwers GY, Rattner DW, Fernandezdel Castillo C: Serous cystadenoma of the pancreas: Tumor growth rates and recommendations for treatment. Ann Surg 2005 242:413-421.

4. Bosman FT, Carneiro F, Hruban RH, Theise ND: WHO Classification of Tumors of the Digestive System. IARC WHO Classification of Tumors;" 43, No3.

5. Compagno J, Oertel JE: Microcystic adenomas of the pancreas(glycogenrich cystadenomas). Am J Clin Pathol 1978, 69:289-298.

6. Compagno J, Oertel JE: Mucinous cystic neoplasms of the pancreas with overt and latent malignancy (cystadenocarcinoma and cystadenoma): A clinicopathological study of 41 cases. Am J Clin Parhol 1978, 69:573-580.

7. Kloppel G, Solcia E, Longnecker DS, Capella C, Sobin LH: Histological typing of tumors of the exocrine pancreas. WHO international histological classification of tumors. 2 edition. Berlin: Springer; 1996

8. George DH, Murphy F, Michalski R, Ulmer BG: Serous cystadenocarcinoma of the pancreas: A new entity? 1989, 13:61-66. 
9. Jonathan CKing, Tina TNg, Stephen CWhite, Cortina Galen, Howard AReber, Joe Hines O: Pancreatic Serous Cystadenocarcinoma: A case report an review of the literature. J Gastrointest Surg 2009, 13:1864-1868.

10. Galanis C, Zamani A, Cameron JL, Campell KA, Lillemoe KD, Caparelli D, Chang D, Hruban RH, Yeo CJ: Resected serous cystic neoplasms of the pancreas: A review of 158 patients with recommendations for treatment. J Gastrointest Surg 2007, 11:820-826.

11. Schmidt-Rohlfing B, Siech M, Mattfeldt T, Schoenberg MH, Bager HG: Cystic neoplasms of the pancreas: surgical treatment and outcome. $Z$ Gastroenterol 1998, 36:939-945.

12. Wu CM, Fisherman EK, Hruban RK: Serous cystic neoplasm involving the pancreas and liver: A unusual clinical entity. Abdom Imaging 1999, 24:75-77.

13. Kimura W, Makuuchi M: Operative indications for cystic lesions of the pancreas with malignant potential: Our experience. Hepatogastroenterology 1999, 46:483-491.

14. Abe H, Kubota K, Mori M, Miki K, Minagawa M, Noise T, Kimura W, Makuutchi M: Serous cystadenoma of the pancreas with invasive growth: Benign or malignant? Am J Gastroenterol 1989, 93:1963-1966.

15. Strobel O, Z'graggen K, Schmitz-Winnenthal FH, Friess H, Kappeler A, Zimmermann A, Uhl W, Buchler MW: Risk of malignancy in serous cystic neoplasms of the pancreas. Digestion 2003, 68:24-33.

16. Friebe V, Keck T, Mattem D, Schmidt-Graeff A, Wemer M, Mikami Y, Adam U, Hopt UT: Serous cystadenocarcinoma of the pancreas: Management of a rare entity. Pancreas 2005, 31:182-187.

17. Verbesey JE, Munson JL: Pancreatic cystic neoplasms. Surg Clin N Am 90 2010, 411-425

18. Federle M, Mc Grath K: Cystic neoplasms of the pancreas. Gastroenterol Clin N Am 36 2007, 365-376.

19. Allen PJ, Jaques DP, D'Angelica M, Bowne WB, Conlon KC, Brennan MF: Cystic lesions of the pancreas: selection criteria for operative and non operative management in 209 patients. J Gastrointest Surg 7 2003, 970-977.

20. Garcea G, Ong SL, Rajesh A, Neal CP, Pollard CA, Berry DP, Dennison AR: Cystic lesions of the pancreas. A diagnostic and management dilemma. Pancreatology 2008, 8(3):236-51.

21. Ferrone CR, Correa-Gallego C, Warshaw AL, Brugge WR, Forcione DG, Thayer SP, Fernandez-del Castillo C: Current trends in pancreatic cystic neoplasms. Arch Surg 2009, 144(5):448-54.

22. McKay A, Sutherland FR, Bathe OF, Dixon E: Morbidity and mortality following multivisceral resections in complex hepatic and pancreatic surgery. J Gastrointest Surg 2008, 12(1):86-90.

doi:10.1186/1477-7819-10-51

Cite this article as: Bramis et al: Serous cystadenocarcinoma of the pancreas: report of a case and management reflections. World Journal of Surgical Oncology 2012 10:51.

\section{Submit your next manuscript to BioMed Central and take full advantage of:}

- Convenient online submission

- Thorough peer review

- No space constraints or color figure charges

- Immediate publication on acceptance

- Inclusion in PubMed, CAS, Scopus and Google Scholar

- Research which is freely available for redistribution 